\title{
Peristeen Transanal Irrigation System to Manage Bowel Dysfunction: A NICE Medical Technology Guidance
}

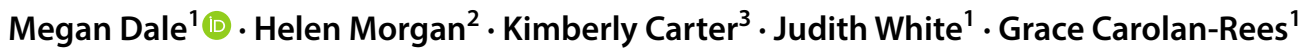

Published online: 14 November 2018

(c) The Author(s) 2018

\begin{abstract}
The Peristeen transanal irrigation system is intended to allow people with bowel dysfunction to flush out the lower part of the bowel as part of their bowel management strategy. Peristeen was the subject of an evaluation by the National Institute for Health and Care Excellence, through its Medical Technologies Evaluation Programme, for the management of bowel dysfunction. The company, Coloplast, submitted a case for adoption of the technology, claiming that the technology improves the severity of chronic constipation or faecal incontinence and improves quality of life for people with bowel dysfunction. Other claimed benefits included reduced frequency of UTIs, stoma surgery and hospitalisation rates, as well as reduced costs. The submission was critiqued by Cedar. The clinical evidence assessed included one randomised controlled trial, and 12 observational studies for adults and 11 studies for children. Although there are limitations in the evidence, the assessed studies show some improvement in outcomes for patients who choose to continue using Peristeen. The committee heard from patient experts that Peristeen had improved their lives and allowed them increased independence. The submitted economic evidence had numerous flaws, however following Cedar's changes to the model, and additional sensitivity analysis, the use of Peristeen was judged unlikely to be cost incurring compared with standard bowel care. The Peristeen transanal irrigation system received a positive recommendation in Medical Technologies Guidance 36.
\end{abstract}

\section{Key Points for Decision Makers}

Peristeen is used by a wide range of people with bowel dysfunction from different causes.

Not all patients find Peristeen acceptable or useful; however, for those who do, it can be a significant improvement in their quality of life and can promote dignity and independence.

Megan Dale

Megan.Dale@wales.nhs.uk

Helen Morgan

MorganHE1@ cardiff.ac.uk

Kimberly Carter

Kimberley.carter@nice.org.uk

Judith White

Judith.White3@wales.nhs.uk

Grace Carolan-Rees

Peristeen can be difficult to use and people may require time, training and support to get comfortable with using it. Some people will choose to stop using Peristeen.

There are considerable uncertainties in the economic evaluation; however, on average, Peristeen is unlikely to cost more than standard bowel care over a relevant time horizon.

Grace.Carolan-Rees@wales.nhs.uk

1 Cedar, Cardiff and Vale University Health Board, Cardiff, UK

2 Cedar, Cardiff University, Cardiff, UK

3 National Institute for Health and Care Excellence, Manchester, UK 


\section{Introduction}

The National Institute for Health and Care Excellence (NICE) produces guidance on new or innovative medical devices or diagnostics-Medical Technologies Guidance (MTG). The aim of the guidance is to support the adoption of clinically effective and cost-saving technologies in the UK National Health Service (NHS). The process for producing MTG has been previously described in detail [1].

This paper summarises Cedar's assessment report and how it was used to inform the NICE MTG on the Peristeen transanal irrigation system to manage bowel dysfunction (MTG36). Cedar is a NHS-academic health care technology research collaboration based in Cardiff. This paper is part of a series that provides an insight into the development of NICE MTG [1].

\section{Background to the Condition and Technology}

Neurogenic bowel dysfunction can be caused by neurological conditions such as spinal cord injury, spina bifida, multiple sclerosis, Parkinson's disease and other conditions associated with impairment or loss of sphincter control and bowel mobility disorders. Bowel dysfunction can also have numerous other causes, such as injury to the rectum or bowel, or slow transit constipation. Symptoms include faecal incontinence and constipation, along with an increased rate of urinary tract infections (UTIs).

Transanal irrigation can be used by people with bowel dysfunction to empty the rectum and some of the colon at a time and frequency that is suitable to them, and to avoid faecal incontinence and constipation.

Peristeen is a transanal irrigation system consisting of a rectal catheter with an inflatable balloon, a manual control unit with a pump, leg straps, and a bag to hold water. The constant flow pump means that the system does not depend on gravity, and the bag does not need to be elevated. A new catheter is required for every use. The manufacturer recommends use every other day, although users will develop their own preference for how frequently to use the irrigation system. Peristeen received a CE mark in May 2003 as a class 1 medical device.

A variety of systems, including Peristeen, are available that differ in design and use. These choices should be discussed by clinician and patient, and a number of systems may be tried before a preferred device for transanal irrigation is found for that particular patient.

Currently, alternative treatment options include medication (oral drugs, suppositories and enemas), changes to diet, and physiotherapy. People with bowel dysfunction may also manage their symptoms using biofeedback, bowel washouts and manual removal of faeces. Some patients may need or may prefer surgery, i.e. a colostomy, ileostomy, sacral nerve stimulation or an antegrade continence enema procedure.

The benefits claimed by the company to people with bowel dysfunction are that use of Peristeen improves symptoms/reduces the severity of chronic constipation; reduces the severity and frequency of faecal incontinence; improves quality of life for people with bowel dysfunction; and reduces the incidence of UTIs in people with neurogenic bowel dysfunction.

The benefits claimed by the company to the healthcare system are that the use of Peristeen reduces the rate of stoma surgery in people with neurogenic bowel dysfunction; reduces the frequency, and therefore the treatment costs, of UTIs; reduces the cost of treating neurogenic bowel dysfunction in people who have experienced unsuccessful standard bowel care as first-line treatment; and reduces the rate of hospitalisation in people with neurogenic bowel dysfunction.

\section{Decision Problem (Scope)}

In their evidence submission, the manufacturer must keep within the scope of the evaluation. The scope is defined by NICE in the form of a PICO table (population, intervention, comparator, outcomes, plus cost analysis and subgroups to be considered).

\subsection{Population}

The population was defined as people with bowel dysfunction in any setting. Although the company claims were based on neurogenic bowel disorder, following consultation NICE broadened the scope to include all bowel dysfunction. Cedar identified that the company had inappropriately excluded most studies on children, and included such studies in their report.

\subsection{Intervention}

The intervention was defined as the Peristeen transanal irrigation system.

\subsection{Comparator}

The comparator was defined as conservative bowel management, which can include treatments such as diet and bowel habit advice, medication, disposable pads and anal plugs, muscle/bowel training, biofeedback and electrostimulation, and digital stimulation and manual evacuation. It was noted 
that the type of treatment is dependent on personal preference, ability and carer support.

\subsection{Outcomes}

The following outcomes were included in the scope: severity and frequency of incontinence, severity of constipation, quality of life, length and frequency of irrigation, devicerelated adverse events, frequency of UTI, incidence of stoma surgery and hospitalisations, staff time (including primary care and community care visits), and individual length of use/user satisfaction.

\section{Cedar's Review of Evidence}

The company provided an evidence submission to NICE that should be in line with the scope. This submission presented the available clinical and cost evidence, alongside a de novo cost model produced by the company. Cedar's assessment report aimed to provide the Medical Technologies Advisory Committee (MTAC) with a balanced, fair, and independent appraisal of the evidence surrounding the use of Peristeen for bowel dysfunction [2]. Broadly, this involved (1) identifying any additional information not included in the manufacturer's submission; (2) appraising the published clinical and cost evidence and, where necessary, presenting additional data; (3) critiquing the de novo cost model by checking inputs, assumptions, and structural integrity; (4) highlighting any key issues; and (5) presenting any new analyses carried out.

\subsection{Review of Clinical-Effectiveness Evidence}

The company submitted 10 studies consisting of 1 randomised controlled trial (RCT) of adults [3], 7 observational studies of adults [4-10], and 1 observational study of children [11], plus 1 prepublication study of adults. Cedar did not agree with the company's justification for excluding all but one paediatric study (because they did not use validated scoring systems) and included all studies with relevant outcomes. Cedar undertook a comprehensive search and selection process (Fig. 1) and included a further 4 observational studies of adults[12-15] and a further 10 observational studies of children [16-25]. The main outcomes in the included studies were patient-reported outcomes, which, due to the nature of the device, was appropriate. Included studies are listed in Table 1.

\subsubsection{Adult Studies}

One reasonable-quality RCT that included 87 adults with spinal cord injuries and neurogenic bowel dysfunction was identified [3]. This unblinded study compared transanal irrigation using Peristeen $(n=42)$ with standard bowel care $(n=45)$ for 10 weeks and reported significant improvements in validated patient-reported outcomes for bowel function. Twelve patients in the Peristeen arm and two standard care patients withdrew from the study. There was a significant improvement in a series of patientreported outcome measures (PROMs)—Cleveland Clinic Constipation Scoring System (CCCS), St Mark's Fecal Incontinence Grading System (FIGS), and Neurogenic Bowel Dysfunction Score (NBDS)-for the Peristeen group compared with standard care (Table 2).

In the observational studies of adults, which reported comparative data (before and after), there was an improvement in patient-reported outcomes for bowel function when using Peristeen. Most of these studies used appropriate standardised PROMs. Outcome measures such as incidence of UTIs and faecal incontinence also improved when using Peristeen, while outcomes related to general quality-of-life measures were less widely reported and changes were either not significant or were significantly improved in only some domains. There were considerable numbers of people who stopped using Peristeen, particularly in the first few months.

\subsubsection{Paediatric Studies}

The paediatric studies were non-comparative, observational, case series. Six of these observational studies were prospective and five were retrospective in design. The studies were of variable quality and used less-consistent outcome measures. General findings indicated the use of Peristeen resulted in improvements in bowel management, however the evidence is weaker than for adults, partly due to the difficulty in obtaining valid PROM data for children.

For both adults and children, there were variable numbers of people who stopped using Peristeen, particularly in the first few months. This was frequently because they disliked using the device or found it painful or ineffective.

\subsubsection{Safety Outcomes}

Bowel perforation is a rare but potentially serious adverse event that can occur with Peristeen use. A global audit [26] based on voluntary reporting to the company found 49 incidents of bowel perforation, 35 of which were certainly caused by Peristeen. Less-serious adverse events include symptoms such as abdominal pain or nausea, which may lead individual patients affected to seek an alternative management strategy. 


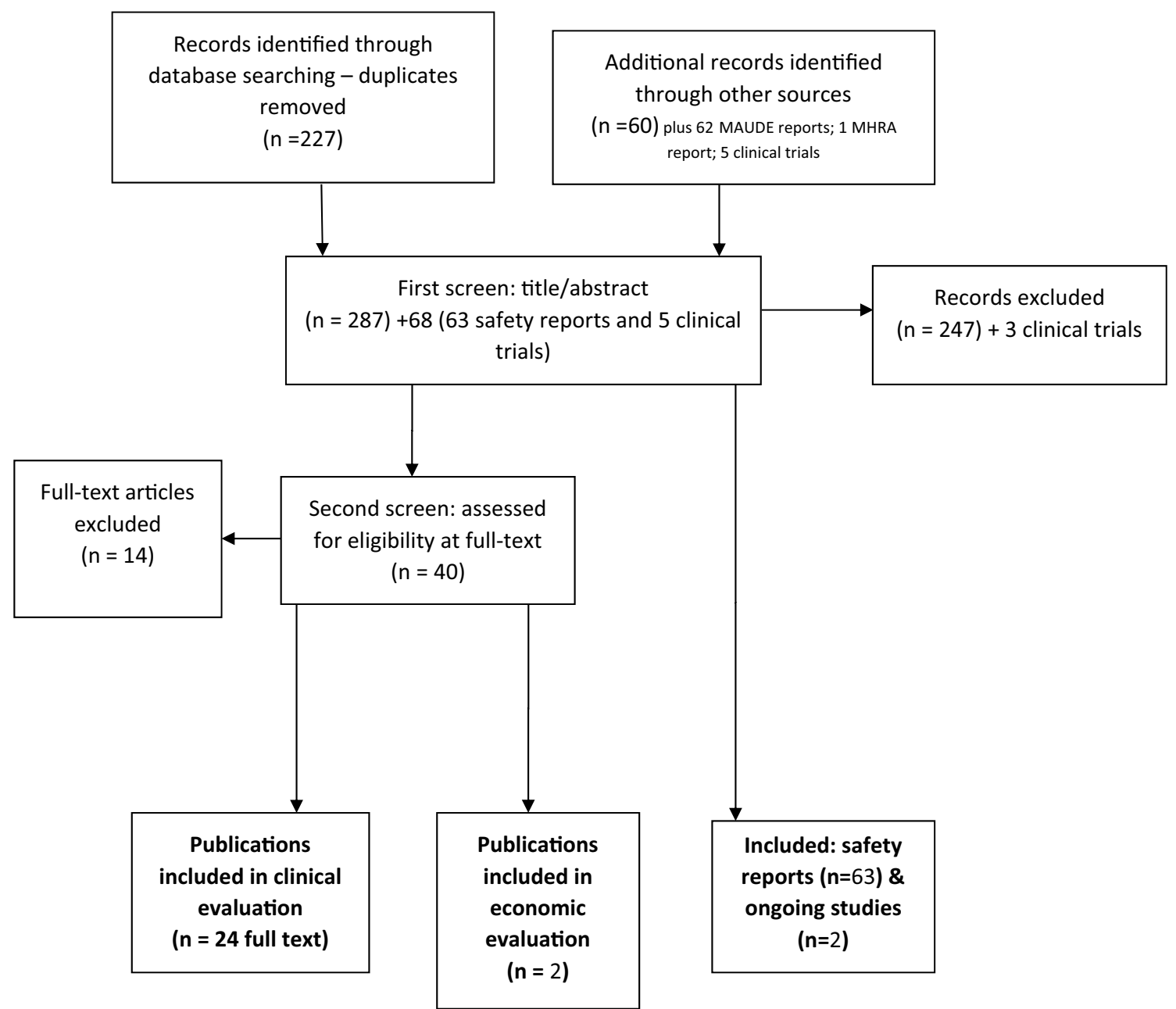

Fig. 1 Cedar's study selection process. MHRA Medicines and Healthcare products Regulatory Agency, MAUDE manufacturer and user facility device experience

\subsection{Review of Economic Evidence}

The company identified two published economic studies [27, 28]. The Christensen et al. [27]. model is from a societal perspective, however it did not match the scope and was therefore excluded by both Cedar and the company. The work of Emmanuel et al. [28]. was the basis for the de novo cost model which is required from the company as part of the evidence submission, and is critiqued in the following sections. The company did not update the costs in the submitted model, but did comment on the impact the updated costs would have.

\subsubsection{Model Structure}

The submitted model was based on the work of Emmanuel et al. [28], a Markov model with a 6-month cycle, comparing the use of the Peristeen transanal irrigation system with standard bowel care for a 30-year-old subject with spinal cord injury. The model time horizon was 37 years, with a discount rate of $3.5 \%$ and an NHS and social services perspective. The available states in the model are management using Peristeen, standard bowel care, surgery, or stoma. Stoma is an end state in this model and patients cannot move from this, as shown in Fig. 2.

Key assumptions in the model are as follows.

- All patients enter the model at age 30 and survive for 37 years. There is no death state in the model.

- The probability of ceasing to use Peristeen is assumed to be constant.

- Adverse events are included as hospital admissions, and are assumed to be split equally between gastrointestinal infections, pressure ulcers, falls or trauma, abdominal pain and UTI. Bowel perforation is not explicitly included.

- The model is stated as being for a patient with spinal cord injuries, and patients are assumed to be homogeneous. 
Table 1 Studies included by both the company and Cedar

\begin{tabular}{|c|c|c|c|c|c|c|}
\hline References & Country & Study type & Population & $n$ & Company & Cedar \\
\hline \multicolumn{7}{|l|}{ Adults } \\
\hline Chan et al. [12] & UK & OBS & Mixed & 91 & - & $\checkmark$ \\
\hline Christensen et al. [4] & Europe including UK & $\mathrm{OBS}^{\mathrm{a}}$ & $\mathrm{SCI}$ & 62 & $\checkmark$ & $\checkmark$ \\
\hline Christensen et al. [3] & Europe, including the UK & RCT & SCI & 87 & $\checkmark$ & $\checkmark$ \\
\hline Del Popolo et al. [5] & Italy & OBS & SCI & 36 & $\checkmark$ & $\checkmark$ \\
\hline \multicolumn{5}{|c|}{ Pre-publication study provided as 'academic in confidence' } & $\checkmark$ & $\checkmark$ \\
\hline Hamonet-Torny et al. [6] & France & OBS & NBD & 16 & $\checkmark$ & $\checkmark$ \\
\hline Kim et al. [13] & South Korea & OBS & SCI & 52 & - & $\checkmark$ \\
\hline Loftus et al. [7] & Ireland & OBS & NBD & 11 & $\checkmark$ & $\checkmark$ \\
\hline Nafees et al. [14] & UK & $\mathrm{DC}$ & Mixed & 129 & & $\checkmark$ \\
\hline Passananti et al. [8] & UK & OBS & MS & 49 & $\checkmark$ & $\checkmark$ \\
\hline Preziosi et al. [9] & UK & OBS & MS & 30 & $\checkmark$ & $\checkmark$ \\
\hline Rosen et al. [10] & $\begin{array}{l}\text { Austria } \\
\text { Switzerland }\end{array}$ & OBS & ARS & 14 & $\checkmark$ & $\checkmark$ \\
\hline Whitehouse et al. [15] & UK & OBS & FBD & 113 & - & $\checkmark$ \\
\hline \multicolumn{7}{|l|}{ Children } \\
\hline Alenezi et al. [16] & Saudi Arabia & OBS & NBD & 18 & $x$ & $\checkmark$ \\
\hline Ausili et al. [17] & Italy & OBS & SB & 60 & $x$ & $\checkmark$ \\
\hline Choi et al. [32] & South Korea & OBS & SB & 44 & $x$ & $x$ \\
\hline Corbett et al. [18] & UK & OBS & Mixed & 24 & $x$ & $\checkmark$ \\
\hline Kelly et al. [19] & USA & OBS & NBD & 24 & $x$ & $\checkmark$ \\
\hline King et al. [20] & Australia & OBS & SB & 20 & $x$ & $\checkmark$ \\
\hline Koppen et al. [21] & Netherlands & OBS & IC & 67 & $x$ & $\checkmark$ \\
\hline Lopez Pereira et al. [22] & Spain & OBS & SB & 40 & $x$ & $\checkmark$ \\
\hline Marzheuser et al. [23] & Germany & OBS & ARM & 40 & - & $\checkmark$ \\
\hline Midrio et al. [11] & Italy & OBS & Mixed & 83 & $\checkmark$ & $\checkmark$ \\
\hline Nasher et al. [24] & UK & OBS & $\mathrm{IC}$ & 13 & $x$ & $\checkmark$ \\
\hline Pacilli et al. [25] & UK & OBS & Mixed & 23 & $x$ & $\checkmark$ \\
\hline
\end{tabular}

${ }^{\mathrm{a}} \mathrm{RCT}$ and OBS paper by Christensen et al. used the same patients

$A R M$ Anorectal malformation, $F B D$ Functional bowel disorder, $M S$ multiple sclerosis, $S B$ spina bifida, $S C I$ spinal cord injury, $N B D$ neurogenic bowel dysfunction, $I C$ idiopathic constipation, $A R S$ anterior resection syndrome, $R C T$ randomised controlled trial, $O B S$ observational, single-arm study, $D C$ discrete choice experiment, $\checkmark$ indicates included, $\boldsymbol{X}$ indicates explicitly excluded
- Transition probabilities and variables are constant over time for all patients. Many variables are likely to change with age for all patients, and will also change over time for patients with progressive diseases such as multiple sclerosis.

- Transition probabilities for patients who start using Peristeen and then revert to standard bowel care are stated in the submission as being the same as probabilities in the standard bowel care arm.

\subsubsection{Data Sources for Outcomes and Resources}

The model uses audit data for 227 patients from three UK hospitals as the main source of clinical and resource data. Data for standard bowel care are taken from the baseline questionnaires for patients, except for the number of patients progressing to stoma, which is taken from the retrospective data of 157 patients prior to the introduction of Peristeen. Limited information on the audit is published [8, 28], but an extract of some information was shared with Cedar by the company. The audit appears to have an appropriate NHS setting, with suitable patient pathways, and an appropriate, if heterogeneous, population. However, Cedar were unable to reconcile differences in the data from the available sources, and had insufficient information available to critique the audit data used in the model.

The statistical techniques used for calculating transition probabilities from patients enrolled at different time points, with different lengths of follow-up, were not stated.

\subsubsection{Data Sources for Adverse Events}

Adverse events are modelled as UTIs that respond to treatment, and events requiring hospitalisation. The 


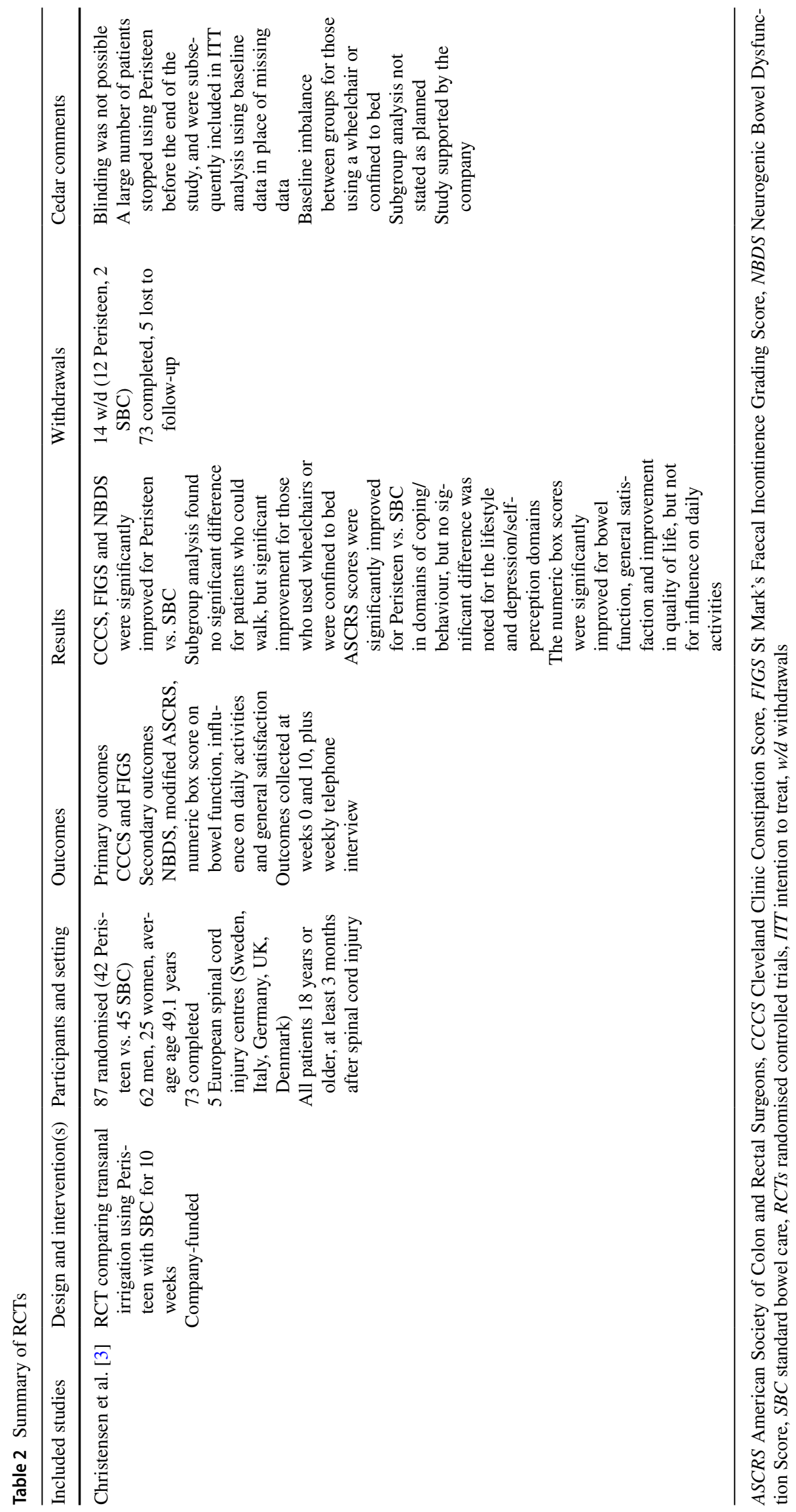


Fig. 2 Markov model structure
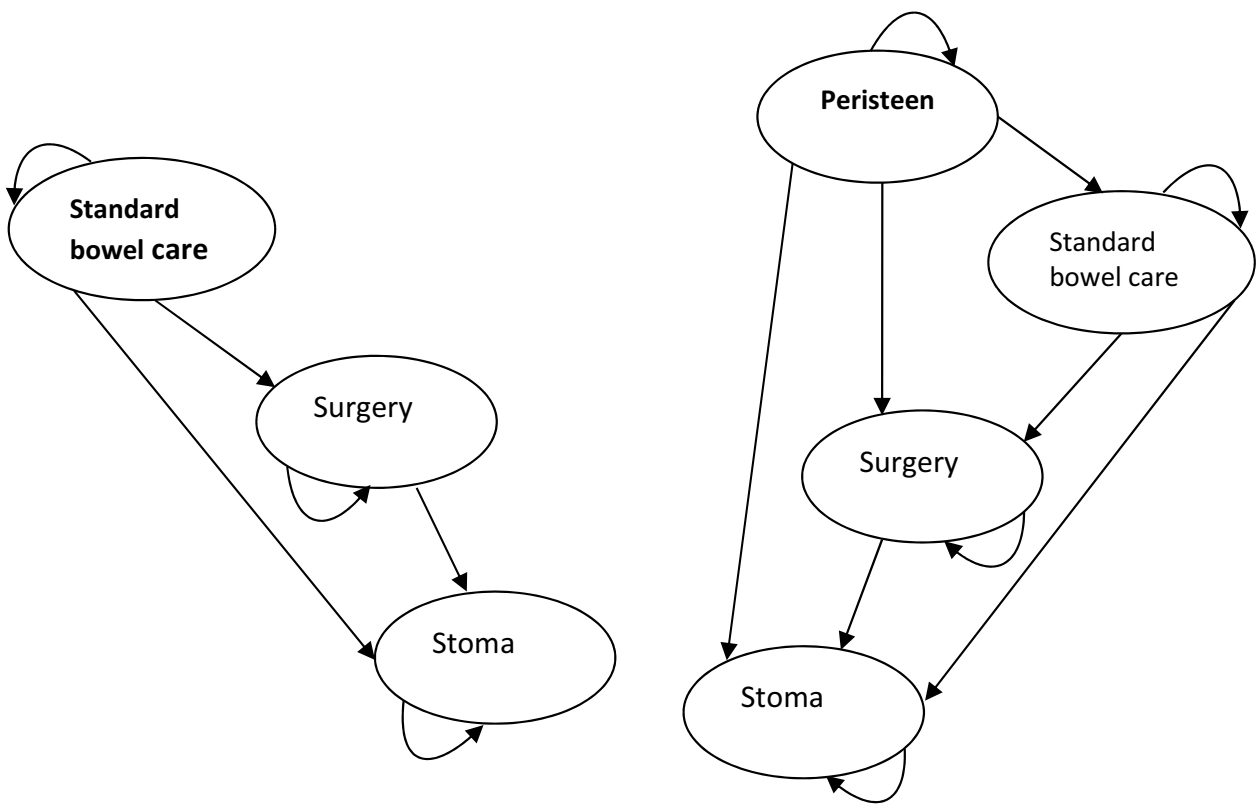

hospitalisations are split equally between abdominal pain, gastrointestinal infection, pressure ulcers, falls and trauma, and UTIs. The assumption of an equal split is not justified and is likely to overestimate treatment for pressure ulcers, which can be very costly.

\subsubsection{Errors}

Cedar identified several errors in the model, the most important of which were all related to people in the Peristeen arm who returned to using standard care. The main errors for these people returning to standard care were:

- the cost of a health care professional's time was not included;

- the cost of standard bowel care-related adverse events was not included;

- there was a lower use of consumables assumed, compared with people in the model who had never used Peristeen;

- a reduced probability of moving to surgery or stoma for people in the model receiving standard bowel care who had previously received Peristeen.

Cedar considered all these points to be modelling errors as they did not reflect the intended model function as described in the company submission. No evidence was submitted to suggest that people who used Peristeen and then changed back to standard care would have any different resource requirement or outcomes compared with those who had never used Peristeen. Once these errors were corrected, the cost saving due to the use of Peristeen was reduced from $£ 21,768$ to $£ 7829$.

\subsubsection{Changes Made by Cedar}

The change made by Cedar that had the largest impact was to change the cost of a pressure ulcer from $£ 24,214$ (Touche Ross [29], inflated) to $£ 15,134$ (Dealy [30], inflated). In addition, Cedar changed the cost of a UTI from $£ 167.77$ per episode to $£ 52.57$ (Berningham [31], inflated) in line with the costs listed in the original study referenced.

In order to reflect the high number of people ceasing to use Peristeen at an early stage, Cedar introduced a variable transition probability. Due to the limited data available, this was set as three different fixed values to empirically recreate the reported dropout rate. Background mortality was also added due to the long time horizon for this model.

The combined impact of these changes further reduced the cost saving to $£ 3176$, as shown in Table 3 .

\subsubsection{One-Way Sensitivity Analysis}

Cedar added frequency of use to the one-way sensitivity analysis and carried out additional sensitivity analysis.

The model is very sensitive to the frequency of use and also sensitive to pressure ulcer treatment and faecal incontinence, and there is uncertainty about each of these variables. There is limited clinical evidence around the frequency of use and for an improvement in faecal incontinence. Furthermore, there is no direct clinical evidence 
Table 3 Impact on the incremental cost of additional work by Cedar

Model version

Incremental

Base-case submitted by the company

$-£ 21,768$

Changes 1-8: correction of errors

$-£ 7829$

Patients in the Peristeen arm returning to SBC have full costs, including appropriate medication, HCP time and adverse events

Carer time in both arms is corrected

Transition probability for all patients receiving SBC is standardised

Changes 9-10: Cedar-suggested refinements

9. Reduced number of Peristeen users in the first year, using variable transition probability

10. Background mortality added

11. Pressure ulcer cost changed to $£ 15,134.84$

12. UTI cost changed to $£ 52.57$

Final Cedar base-case with all corrections and refinements

$S B C$ standard bowel care, $H C P$ healthcare professional, $U T I$ urinary tract infection

linking the use of Peristeen to a reduction in pressure ulcers.

\subsubsection{Key Drivers}

A key driver in the model is the frequency of use, due to the need for a new catheter at each use. The average frequency is likely to be correctly modelled every other day, however the actual use for each patient may vary from daily to weekly depending on their preferences and circumstances.

The cost saving identified in Cedar's corrected model in the Peristeen arm is largely due to reduced time for health care professional visits and carer time; reduced incidence of faecal incontinence (requiring fewer incontinence pads); and reduced incidence of UTIs and fewer hospitalisations.

\section{National NICE Guidance}

\subsection{Preliminary Guidance}

The evidence submitted by the company and Cedar's report were presented to the MTAC, who produced the following draft recommendations:

The case for adopting Peristeen for managing neurogenic bowel dysfunction in adults is supported by the evidence. Peristeen can reduce the severity of constipation and incontinence, improve bowel-related quality of life, and promote dignity and independence.

Peristeen may not be suitable for all people with neurogenic bowel dysfunction and can be difficult to use. It may take several weeks before a person is comfortable using it themselves, and some people may choose to stop using it.
Peristeen is therefore most effective when it is offered with specialist training for users, carers and NHS staff, along with structured patient support.

Cost modelling for Peristeen is associated with significant uncertainties, but it is likely that, overall, Peristeen provides additional clinical benefits without costing more than standard bowel care.

\subsection{Consultation Response}

During the consultation period, NICE received 43 consultation comments from 18 consultees ( 9 NHS professionals, 1 private sector healthcare professional, 1 professional society, 1 patient group and 6 manufacturers). The comments related to Peristeen use in children, functional bowel disorders, user experience, other transanal irrigation devices, frequency of use, cost and adverse events. New information was submitted from centres using Peristeen in children and in people of all ages with functional bowel disease. This information was reviewed by Cedar and presented to the committee. Because of this, the committee broadened the recommendations to include people with bowel dysfunction of any age.

\section{Key Challenges and Learning Points}

The Peristeen transanal irrigation system can be used successfully by a wide range of people whose bowel dysfunction may have different causes and symptoms. This leads to a very broad scope, and the studies that inform the clinical evidence have diverse populations and outcomes. This breadth and diversity mean that combining the clinical evidence presents particular challenges. In addition, the outcomes 
most important to the people who use Peristeen may not be the outcomes that are most easily captured and reported in clinical studies.

The economic evaluation aspect presented significant challenges due to the lack of transparency in the data used for clinical and resource inputs, and the significant structural errors contained in the model. The focus of the sensitivity analysis on costs rather than resource use, and the omission of key factors, such as frequency of use, was potentially misleading.

\section{Conclusions}

The Peristeen transanal irrigation system received a positive recommendation from NICE and should be considered as an option for people requiring additional treatment strategies to manage bowel dysfunction. Published evidence showed that the Peristeen transanal irrigation system improved patient-reported outcomes in those patients who continued to use it. The evidence is more robust for the adult population, partially due to the availability of appropriate validated outcome measures. Consultation responses reported the successful use of Peristeen in children in the UK. The submitted economic evidence was based on audit data that were not available for scrutiny, and the model contained several errors. Despite the uncertainty that remains in the model, it is likely that use of the Peristeen transanal irrigation system will not cost any more than standard care.

Acknowledgements Shaun Harris (Swansea Centre for Health Economics, Swansea University) provided advice on the health economic analysis; Joelle Williams and Robert Palmer (Cedar, Cardiff and Vale University Health Board) assisted in inputs and checking for economic modelling; and Andrew Cleves and Ruth Poole (Cedar, Cardiff and Vale University Health Board) assisted in the preparation of the assessment report. The authors thank Miss Karen Nugent (Consultant, Pelvic Floor and Colorectal Surgery, University Hospital Southampton), Professor Anton Emmanuel (University College London), Mr. Oliver Jones (Consultant, Colorectal Surgery, Oxford University Hospital) and Dr Ian Beales (Consultant, Gastroenterology, Norfolk and Norwich University Hospital) for providing expert clinical advice.

Author Contributions MD, JW, HM, KC and GCR contributed to the preparation of this article. GCR reviewed the full assessment report, as well as this article, and acts as a guarantor for the overall content.

Compliance with Ethical Standards Cedar were funded for their work by the NICE Medical Technologies Evaluation Programme. MD, JW and GCR are NHS employees, and the NHS has a financial interest in the guidance on which this project is based. HM is a Cardiff University employee and has no conflicts of interest to declare. KC is a NICE employee and had no role in the production of the assessment report, but contributed to the preparation of this article. This summary of the Medical Technology Guidance was produced following publication of the final guidance report. The article has not been externally peer reviewed by Applied Health Economics and Health Policy.

Open Access This article is distributed under the terms of the Creative Commons Attribution-NonCommercial 4.0 International License (http://creativecommons.org/licenses/by-nc/4.0/), which permits any noncommercial use, distribution, and reproduction in any medium, provided you give appropriate credit to the original author(s) and the source, provide a link to the Creative Commons license, and indicate if changes were made.

\section{References}

1. Campbell B, Campbell M. NICE medical technologies guidance: a novel and rigorous methodology to address a new health technology assessment challenge. Appl Health Econ Health Policy. 2012;10(5):295-7.

2. Dale M, Ray A, Morgan H, Poole R, Carolan-Rees G. External assessment centre report: peristeen anal irrigation system to manage bowel dysfunction. NICE commissioned report 2017. https ://www.nice.org.uk/guidance/gid-mt511/documents/assessment -report-2. Accessed 8 Oct 2018.

3. Christensen P, Bazzocchi G, Coggrave M, Abel R, Hultling C, Krogh K, et al. A randomized, controlled trial of transanal irrigation versus conservative bowel management in spinal cord-injured patients. Gastroenterology. 2006;131(3):738-47.

4. Christensen P, Bazzocchi G, Coggrave M, et al. Outcome of transanal irrigation for bowel dysfunction in patients with spinal cord injury. J Spinal Cord Med. 2008;31(5):560-7.

5. Del Popolo G, Mosiello G, Pilati C, Lamartina M, Battaglino F, Buffa $P$, et al. Treatment of neurogenic bowel dysfunction using transanal irrigation: a multicenter Italian study. Spinal Cord. 2008;46(7):517-22.

6. Hamonet-Torny J, Bordes J, Daviet JC, Dalmay F, Joslin F, Salle JY. Long-term transanal irrigation's continuation at home. Preliminary study. Ann Phys Rehabil Med. 2013;56(2):134-42.

7. Loftus C, Wallace E, McCaughey M, Smith E. Transanal irrigation in the management of neurogenic bowel dysfunction. Ir Med J. 2012;105(7):241-3.

8. Passananti V, Wilton A, Preziosi G, Storrie JB, Emmanuel A. Long-term efficacy and safety of transanal irrigation in multiple sclerosis. Neurogastroenterol Motil. 2016;28(9):1349-55.

9. Preziosi G, Gosling J, Raeburn A, et al. Transanal irrigation for bowel symptoms in patients with multiple sclerosis. Dis Colon Rectum. 2012;55(10):1066-73.

10. Rosen H, Robert-Yap J, Tentschert G, Lechner M, Roche B. Transanal irrigation improves quality of life in patients with low anterior resection syndrome. Colorectal Dis. 2011;13(10):e335-8.

11. Midrio P, Mosiello G, Ausili E, Gamba P, Marte A, Lombardi L, et al. Peristeen ${ }^{\circledR}$ transanal irrigation in paediatric patients with anorectal malformations and spinal cord lesions: a multicentre Italian study. Colorectal Dis. 2016;18(1):86-93.

12. Chan DS, Saklani A, Shah PR, Lewis M, Haray PN. Rectal irrigation: a useful tool in the armamentarium for functional bowel disorders. Colorectal Dis. 2012;14(6):748-52.

13. Kim HR, Lee BS, Lee JE, Shin HI. Application of transanal irrigation for patients with spinal cord injury in South Korea: a 6-month follow-up study. Spinal Cord. 2013;51(5):389-94.

14. Nafees B, Lloyd AJ, Ballinger RS, Emmanuel A. Managing neurogenic bowel dysfunction: what do patients prefer? A discrete choice experiment of patient preferences for transanal irrigation and standard bowel management. Patient Pref Adherence. 2016;10:195-204. 
15. Whitehouse PA, McWilliams D, Katt C, et al. Peristeen rectal irrigation for functional bowel disorders: which patients benefit? Gastrointest Nurs. 2010;8(2):40-6.

16. Alenezi H, Alhazmi H, Trbay M, et al. Peristeen anal irrigation as a substitute for the MACE procedure in children who are in need of reconstructive bladder surgery. J Can Urol Assoc. 2013;8(1-2):E12-5.

17. Ausili E, Focarelli B, Tabacco F, et al. Transanal irrigation in myelomeningocele children: an alternative, safe and valid approach for neurogenic constipation. Spinal Cord. 2010;48(7):560-5.

18. Corbett P, Denny A, Dick K, et al. Peristeen integrated transanal irrigation system successfully treats faecal incontinence in children. J Pediatr Urol. 2014;10(2):219-22.

19. Kelly M, Dorgalli C, McLorie G, Khoury A. Prospective evaluation of Peristeen transanal irrigation system with the validated neurogenic bowel dysfunction score sheet in the pediatric population. Neurourol Urodyn. 2017;36(3):632-5.

20. King SK, Stathopoulos L, Pinnuck L, Wells J, Hutson J, Heloury Y. Retrograde continence enema in children with spina bifida: not as effective as first thought. J Paediatr Child Health. 2017;53(4):386-90.

21. Koppen IJN, Kuizenga-Wessel S, Voogt HW, et al. transanal irrigation in the treatment of children with intractable functional constipation. J Pediatr Gastroenterol Nutr. 2017;64(2):225-9.

22. Lopez Pereira P, Salvador OP, Arcas JA, et al. Transanal irrigation for the treatment of neuropathic bowel dysfunction. J Pediatr Urol. 2010;6(2):134-8.

23. Marzheuser S, Karsten K, Rothe K. Improvements in incontinence with self-management in patients with anorectal malformations. Eur J Pediatr Surg. 2016;26(2):186-91.
24. Nasher O, Hill R, Peeraully R, et al. Peristeen transanal irrigation system for paediatric faecal incontinence: a single centre experience. Int J Pediatr. 2014;2014:954315.

25. Pacilli M, Pallot D, Andrews A, et al. Use of Peristeen transanal colonic irrigation for bowel management in children: a singlecenter experience. J Pediatr Surg. 2014;49(2):269-72.

26. Christensen P, Andreasen J, Ehlers L. Global audit on bowel perforations related to transanal irrigation. Techn Coloproctol. 2016;20(2):109-15.

27. Christensen P, Andreasen J, Ehlers L. Cost-effectiveness of transanal irrigation versus conservative bowel management for spinal cord injury patients. Spinal Cord. 2009;47(2):138-43.

28. Emmanuel A, Kumar G, Christensen P, et al. Long-term costeffectiveness of transanal irrigation in patients with neurogenic bowel dysfunction. PLoS One. 2016;11(8):e0159394.

29. Touche Ross and Co. The cost of pressure sores. London: Touche Ross and Co.; 1993.

30. Dealey C, Posnett J, Walker A. The cost of pressure ulcers in the United Kingdom. J Wound Care. 2012;21(6):261-6.

31. Bermingham SL, Hodgkinson S, Wright S, Hayter E, Spinks J, Pellowe C. Intermittent self catheterisation with hydrophilic, gel reservoir, and non-coated catheters: a systematic review and cost effectiveness analysis. BMJ. 2013;346:e8639.

32. Choi EK, Han SW, Shin SH, Ji Y, Chon J, Im Y. Long-term outcome of transanal irrigation for children with spina bifida. Spinal Cord. 2015;53:216-20. 